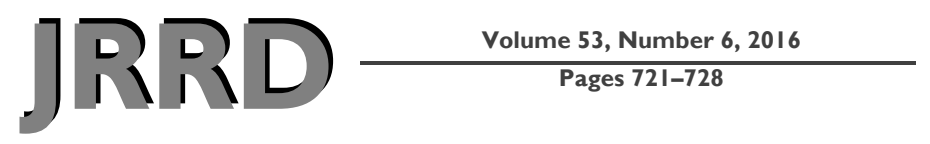

\title{
Prosthesis management of residual-limb perspiration with subatmospheric vacuum pressure
}

\author{
Glenn K. Klute, PhD; ${ }^{1-2^{*}}$ Kathleen J. Bates, MS; ${ }^{1}$ Jocelyn S. Berge, MSE; ${ }^{1}$ Wayne Biggs, CPO; ${ }^{1}$ Charles King, CP$^{3}$ \\ ${ }^{1}$ Center of Excellence for Limb Loss Prevention and Prosthetic Engineering, Rehabilitation Research and Develop- \\ ment, Department of Veterans Affairs Puget Sound Health Care System, Seattle, WA; ${ }^{2}$ Department of Mechanical Engi- \\ neering, University of Washington, Seattle, WA; ${ }^{3}$ Arusha Control Inc, Cumberland, MD
}

\begin{abstract}
For the ambulatory person with lower-limb amputation, insufficient management of perspiration can result in inadequate prosthesis adherence, reduced mobility, and discomfort. This study compared a dynamic air exchange (DAE) prosthesis designed to expel accumulated perspiration with a total surface bearing suction socket (Suction) that cannot. Five people with unilateral transtibial amputation participated in a randomized, crossover experiment. All subjects were given a $1 \mathrm{wk}$ acclimation period to each study prosthesis while we measured their step activity levels. A rest-walk-rest protocol, including a 30 min treadmill walk at a self-selected speed while wearing thermally insulative garments, was then used to observe residual-limb skin temperatures and perspiration. Afterward, subject opinions about the prostheses were assessed with questionnaires. During the weeklong acclimation period, no statistical difference in step activity levels were detected between prostheses ( $p=0.22$ ), but this may have been due to self-reported behavioral modifications. During the rest-walkrest protocol, no differences in skin temperatures were observed $(p=0.37)$. The DAE prosthesis accumulated $1.09+/-$ $0.90 \mathrm{~g}$ and expelled $0.67+/-0.38 \mathrm{~g}$ of perspiration, while the Suction prosthesis accumulated $0.97+/-0.75 \mathrm{~g}$. The questionnaire results suggest that participants were receptive to both prostheses. The DAE prosthesis was able to expel more than a third of the total perspiration, suggesting it may enable longer uninterrupted periods of perspiration-inducing activity.
\end{abstract}

Key words: activity level, amputation, comfort, dynamic air exchange, lower limb, perspiration, prosthesis, skin temperature, suction, sweat.

\section{INTRODUCTION}

People with lower-limb amputation often complain about uncomfortable residual-limb skin temperatures and the accumulation of perspiration within their prostheses [1-6]. Even short bouts of walking can cause substantial increases in residual-limb skin temperatures [7-8] and result in cumulative increases throughout the course of a typical day [9]. The physiological response to an increase in activity can include both vasodilation and sympathetic stimulation of the residual limb's sweat glands [10]. A little moisture can actually increase the skin coefficient of friction [11-12], but if perspiration continues, a threshold is exceeded [13-14] where adherence of the prosthesis to the residual limb becomes insecure. Many people with amputation can sense the impending loss of adherence during vigorous activities and are able to stop, doff the prosthesis, wipe it and the limb dry, and then don it again. However, some circumstances may not afford such accommodations, or it may be socially undesirable to do so.

\footnotetext{
Abbreviations: $\mathrm{DAE}=$ dynamic air exchange, $\mathrm{PEQ}=$ Prosthesis Evaluation Questionnaire, VA $=$ Department of Veterans Affairs.

*Address all correspondence to Glenn K. Klute, PhD; 1660 S. Columbian Way, Seattle, WA 98108; 206-277-6724; Email: gklute@u.washington.edu http://dx.doi.org/10.1682/JRRD.2015.06.0121
} 
One approach to maintaining secure adherence under perspiration-inducing conditions is to keep skin temperatures below where perspiration begins. Webber and Davis incorporated a helical channel in the socket wall to cool the residual limb [15]. Ghoseiri et al. constructed a socket with a thermoelectric heat pump coupled to an aluminum heat-transfer structure integral with the socket wall [16]. Han et al. used heat pipes to concentrate heat flux to a compact heat sink where a fan could convect heat from the prosthesis to the surroundings [17]. Wernke et al. tested a liner with phase change material and found residual-limb skin temperatures to be lower at the end of an exercise bout and, for patients that perspired, that less accumulation occurred than with a liner without phase change materials [18].

An alternative approach is to expel perspiration from the prosthesis as it accumulates. This design transports perspiration by means of a miniature pump and solenoid airflow control system that can create a small pressure differential (vacuum or negative gauge pressure) between the proximal and distal regions of the donned prosthesis. This pressure differential, when carefully controlled, can be used to cause airflow (dynamic air exchange) between the prosthetic liner and the skin. This air flow can provide the means for expelling perspiration into an exterior reservoir and may also provide evaporative cooling of the residual limb.

To compare the performance of this novel prosthesis with a standard-of-care prosthesis (total surface bearing suction socket), we conducted a within-subject crossover experiment to measure differences in (1) activity levels, (2) residual-limb skin temperatures, (3) perspiration accumulation by both prostheses and expulsion by the novel prosthesis, and (4) subjective experiences.

\section{METHODS}

Moderately active people with unilateral transtibial amputation were recruited to participate in this institutional review board-approved study. Participants were between the age of 18 and $70 \mathrm{yr}$, wore their prosthesis at least $4 \mathrm{~h} / \mathrm{d}$, were at least 6 mo postamputation, were able to walk at a steady pace for at least $30 \mathrm{~min}$ on a treadmill, and did not have a dysvascular condition or diabetes. Participants were also able to detect the touch of a SemmesWeinstein 5.07 monofilament (10 g force; CHS Services Inc; East Setauket, New York), used to make skin con- tact, bend, and depart the skin at each temperature sensor site and the proximal edge of the moisture-wicking sock with airflow seal that encircled the limb while wearing one of the study prostheses.

Two study prostheses were worn by each participant: a novel, dynamic air exchange (DAE) prosthesis and a standard-of-care (Suction) prosthesis. Both were fit by a certified prosthetist. The DAE prosthesis (Figure 1) included a custom moisture-wicking textile sock with a proximal elastomeric airflow seal (Figure 2); a modifiedpin lock elastomeric liner (Figure 3); and a total contact socket with a custom-designed, battery-operated negative gauge pressure-generating device and associated components attached to the socket exterior. The DAE system uses a small pump (CTS Series, Parker Hannifin; Hollis, New Hampshire) in fluid communication with occlusionpreventing liner ports (four proximal, one distal) to obtain a small proximal-to-distal pressure differential ( $\sim 10 \mathrm{kPa}$ ) across the residual limb for secure adherence. A solenoid valve (High Density Interface solenoid valve, The Lee Company; Westbrook, Connecticut), which can be opened on demand by the user, allowed air flow through the sock weave from proximal to distal, ventilating the subject's skin. Depending on fit, the air flow may be up to $1.4 \mathrm{~L} / \mathrm{min}$. The pressure differential also enabled expulsion of perspiration accumulating at the distal end of the residual limb. The Suction prosthesis included an elastomeric liner, a total surface bearing suction socket with an expulsion valve, and an elastomeric sleeve. For both systems, a passive energy-storing prosthetic foot (LP Vari-Flex with EVO, Össur; Foothill Ranch, California) with aluminum pylon was attached to the socket. When needed, an additional wool or synthetic sock was worn exterior to the liner to improve socket fit.

Participants were randomly assigned to begin the study with either the DAE or Suction prosthesis, fit with a step activity monitor (StepWatch3, Orthocare Innovations; Mountlake Terrace, Washington) to measure their activity levels, and given a 1 wk acclimation period before they returned to the laboratory for a $2 \mathrm{~h}$ assessment. Subjects were then switched to the other study prosthesis, given a second $1 \mathrm{wk}$ acclimation period, and again returned to the laboratory for a $2 \mathrm{~h}$ assessment.

During the laboratory assessment, subjects were asked to change into a polypropylene insulating layer (long-sleeved shirt and shorts), a polar fleece pull-over, a polar fleece cap, and knee length socks. All prosthetic components with potential to accumulate moisture were 

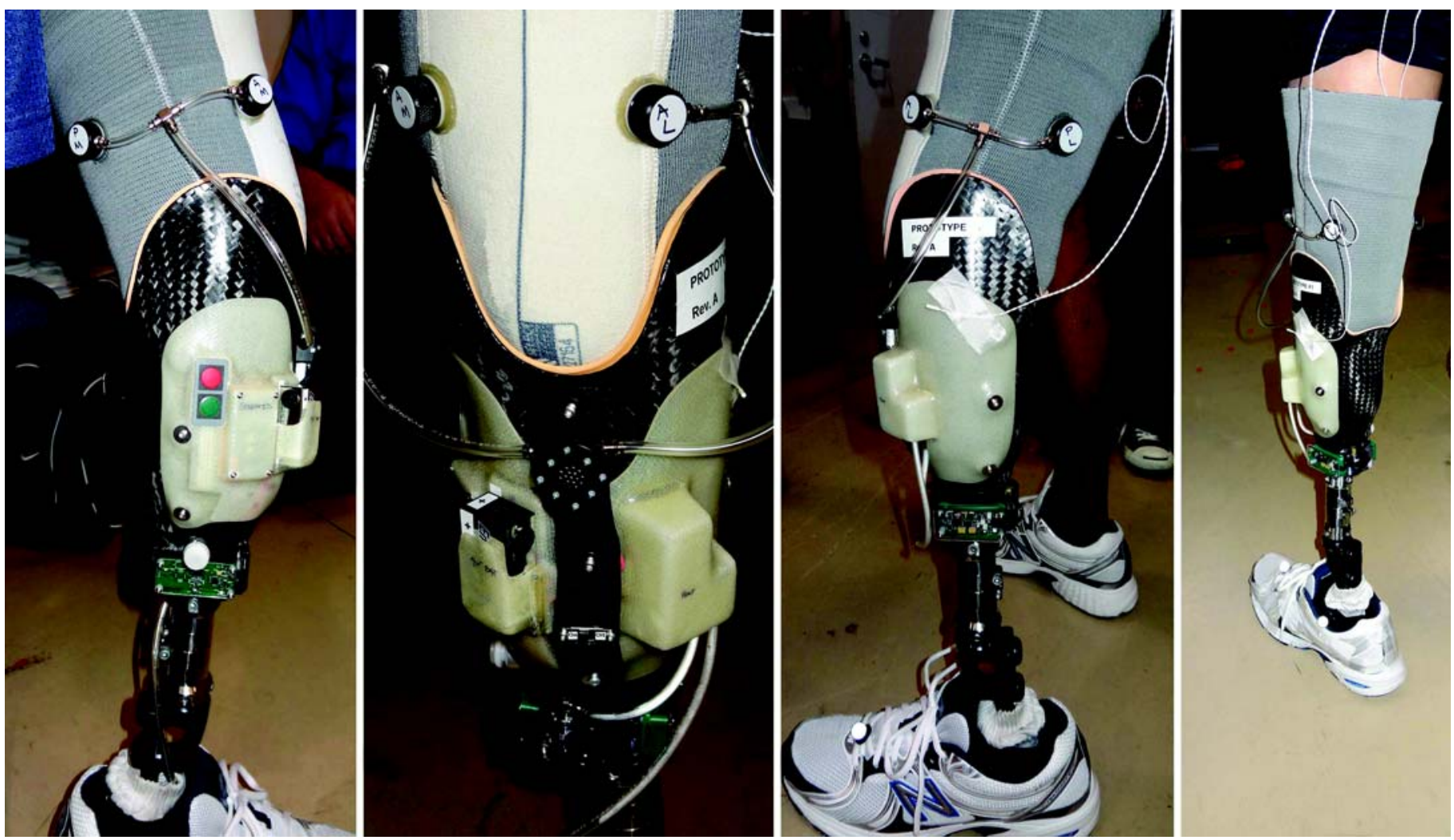

Figure 1.

Medial, anterior, lateral, and posterior views (from left to right) of the dynamic air exchange prosthesis.

weighed. Two small thermistors (model MA 100BF, Thermometrics; Edison, New Jersey), powered and sampled at $0.125 \mathrm{~Hz}$ with a portable data acquisition unit (SmartReader Plus 8, ACR Systems Inc; Surrey, Canada), were taped to the residual-limb skin over the medial and lateral borders of the gastrocnemius muscle. The paired thermistor wires were routed up the limb on the medial and lateral sides. The prosthesis was donned over the thermistors.

Each subject then rested while seated for $30 \mathrm{~min}$, walked at self-selected speed on a treadmill (Bertec Instrumented Treadmill, Bertec Corporation; Columbus, Ohio) for $30 \mathrm{~min}$, and then rested again while seated for 30 min. For the session with the DAE prosthesis, subjects were asked to activate the dynamic air exchange function at the beginning of the exercise bout and have it remain on until the completion of the second rest period. After the completion of the rest-walk-rest sequence, the subject's prosthesis and the inside of the liner were wiped down with paper towels and weighed along with all com- ponents that could accumulate moisture. The change in residual-limb skin temperature, maximum minus minimum observed during each 30 min period (rest, walk, rest), was calculated for each sensor site for both study prostheses.

Subjective experiences were assessed with two questionnaires at the end of each laboratory assessment. The first questionnaire was the Prosthesis Evaluation Questionnaire (PEQ) [19]. This standardized, self-report instrument is specific to persons with lower-limb amputations and is used to evaluate prosthetic care with regard to prescription and prosthesis-related quality of life. Three scales measuring ambulation, frustration, and residual-limb health were scored. The ambulation questions queried ability to walk in general, in close spaces, on stairs and ramps, in urban environments, and on slippery surfaces. Frustration was assessed by frequency of occurrence and rating. Residual-limb health questions examined sweat, smell, volume changes, rashes, ingrown hairs, and blisters. All three scales were scored so that 


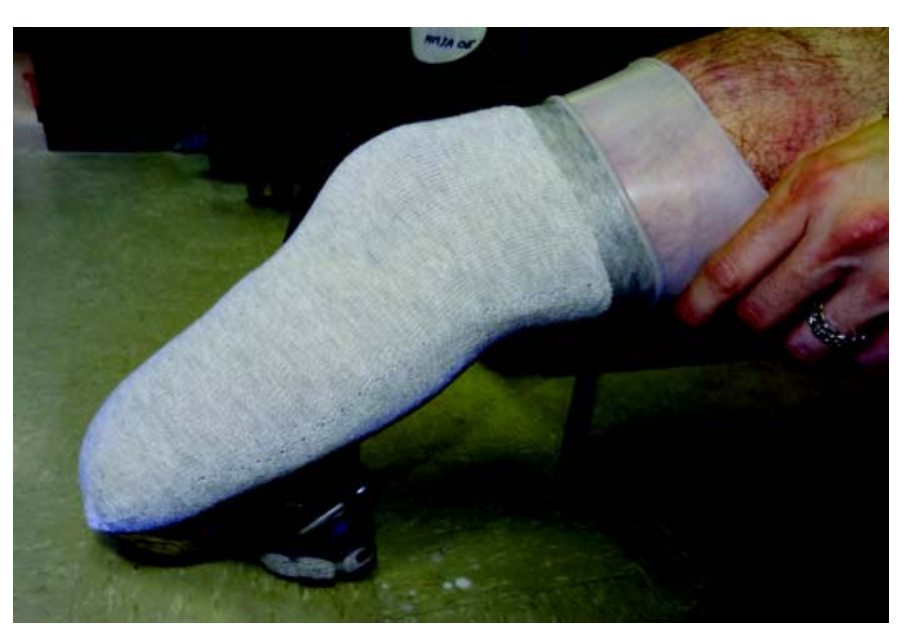

Figure 2.

Moisture-wicking sock shown with proximal elastomeric airflow seal.

100 indicated the best outcome (i.e., most healthful, easiest to walk on, least frustrating). The questions were modified from the original, which uses a 1 mo period of experiences upon which to base subjective answers, to a $1 \mathrm{wk}$ period of experiences.

The second questionnaire was a custom, self-report questionnaire consisting of five questions to assess the prostheses' thermal and moisture management acceptability. The questions were scored from strongly disagree (0) to strongly agree (10) and included (1) "My residual limb gets too hot and sweaty when I am active in this socket system," (2) "I find this socket system keeps my residual limb at a very comfortable temperature,” (3) "My prosthesis feels like it is sliding up and down or falling off when I am active," (4) "I have been more active than normal as a result of this prosthesis," and (5) "I have to stop and dry my residual limb when wearing this prosthesis.”

A paired $t$-test was used to determine whether differences in step activity levels were statistically significant $(p<0.05)$ between the DAE and Suction prostheses. A linear mixed model was used to assess whether differences in residual-limb skin temperatures were statistically significant $(p<0.05)$ by prosthesis and sensor site. Exact $p$-values are reported for each test. The analysis was conducted using SPSS version 19 (IBM Corporation; Armonk, New York). Accumulation of perspiration, expulsion of perspiration (DAE prosthesis only), and subjective experiences were not statistically analyzed because of large expected variances.

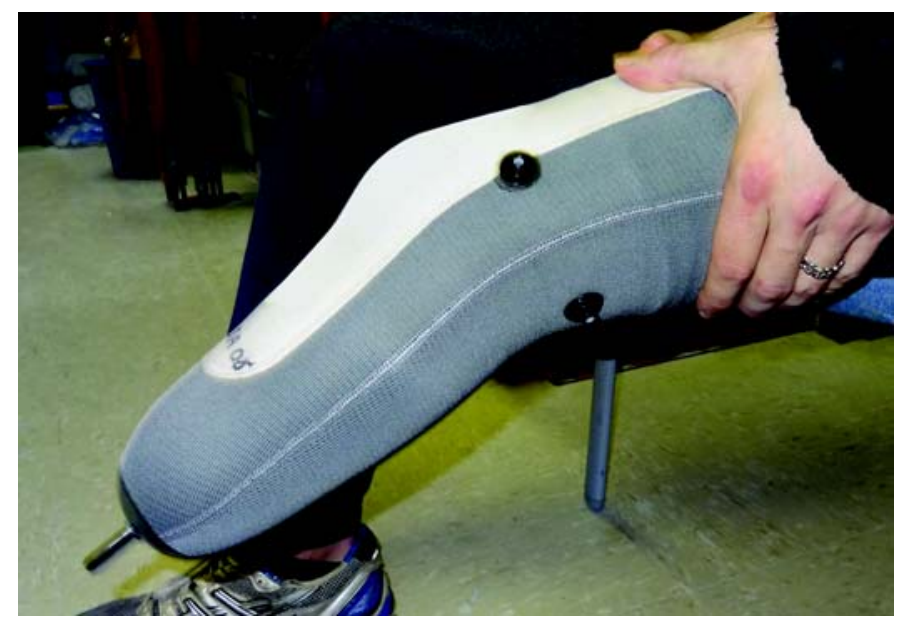

Figure 3.

Prosthetic liner with proximal and distal (through the hollow locking pin) air flow ports.

\section{RESULTS}

The sample population of this study consisted of moderately active community ambulators who might benefit from improved perspiration management. Seven males with transtibial, unilateral amputation provided informed consent. Two subjects were withdrawn after providing informed consent but before completing any study procedures: one who became unreachable and one because of concerns about obesity and structural safety margins of the novel prosthesis. Four completed the protocol as planned, and one completed the protocol with a minor deviation. The subject who deviated experienced a weight gain and had fit issues half way through the protocol. This subject was fit with a larger prosthesis and then completed the remainder of the protocol. The five participants who completed the protocol were $44 \pm 15$ yr old (mean \pm standard deviation), $14 \pm 15$ yr postamputation (3 traumatic etiology and 2 secondary to infection), with a height of $1.83 \pm 0.05 \mathrm{~m}$ and a body mass of $89 \pm 18 \mathrm{~kg}$.

Observations of their activity levels over the $1 \mathrm{wk}$ acclimation period found subjects were not significantly more active ( $p=0.22)$ while wearing the Suction prosthesis $(3,649 \pm 2,411$ prosthetic leg steps/d) than while wearing the DAE prosthesis $(2,607 \pm 2,308$ prosthetic leg steps/d).

There were no differences in residual-limb skin temperature across study prosthesis ( $p=0.37$ ) or sensor site ( $p=0.68$ ) during the rest-walk-rest protocol while wearing 
thermally insulative clothing. The residual-limb skin temperature generally stayed constant during the first 30 min rest period, increased $\left(\sim 3^{\circ} \mathrm{C}\right)$ during the $30 \mathrm{~min}$ walk at self-selected speed, and fell gradually $\left(\sim 1^{\circ} \mathrm{C}\right)$ during the final 30 min rest period (Table).

After the rest-walk-rest protocol when subjects were wearing thermally insulative clothing, our observations found that the DAE prosthesis accumulated $1.09 \pm 0.90 \mathrm{~g}$ and expelled $0.67 \pm 0.38 \mathrm{~g}$ perspiration, while the Suction prosthesis accumulated $0.97 \pm 0.75 \mathrm{~g}$. Neither limb lost adherence during the test, nor did any of the subjects stop walking because of adherence (or any other) concerns.

As measured by the PEQ, subjects opined that their residual limb was healthier while wearing the DAE ( $89 \pm$ $15)$ than with the Suction ( $66 \pm 30)$; it was easier to ambulate while wearing the DAE $(76 \pm 25)$ than with the Suction (65 \pm 32$)$, but wearing the DAE $(49 \pm 32)$ was more frustrating than the Suction (58 \pm 40 ).

As measured by the custom questionnaire, subjects agreed $(7.6 \pm 1.8)$ that the Suction prosthesis got too hot and sweaty during activity but strongly disagreed ( $0.8 \pm$ 1.0) when wearing the DAE prosthesis. Subjects slightly disagreed $(4.0 \pm 3.2)$ that the Suction prosthesis kept their residual limb at a very comfortable temperature, but they strongly agreed $(8.8 \pm 0.6)$ with the statement when wearing the DAE prosthesis. With regard to adherence, subjects somewhat disagreed that both the Suction prosthesis and the DAE prosthesis $(3.0 \pm 3.7$ vs $3.0 \pm 2.4$, respectively) felt insecure during activity. Subjects slightly disagreed $(3.7 \pm 3.4)$ that they were more active than normal when wearing the Suction prosthesis, and slightly agreed $(5.6 \pm 2.1)$ that they were more active

\section{Table.}

Change in residual-limb skin temperature (mean \pm standard deviation) while wearing study prostheses during first rest period, exercise period, and second rest period.

\begin{tabular}{lcc}
\hline Time Period & DAE $\left({ }^{\circ} \mathbf{C}\right)$ & Suction $\left({ }^{\circ} \mathbf{C}\right)$ \\
\hline First Rest & $0.0 \pm 0.4$ & $0.0 \pm 0.3$ \\
$\quad$ Medial Gastrocnemius & $0.0 \pm 0.3$ & $0.5 \pm 0.3$ \\
$\quad$ Lateral Gastrocnemius & $3.1 \pm 0.8$ & $3.4 \pm 0.7$ \\
Exercise & $2.8 \pm 0.4$ & $2.9 \pm 0.8$ \\
$\quad$ Medial Gastrocnemius & & \\
$\quad$ Lateral Gastrocnemius & $-1.1 \pm 0.2$ & $-1.1 \pm 0.1$ \\
Second Rest & $-0.9 \pm 0.5$ & $-0.8 \pm 0.3$ \\
$\quad$ Medial Gastrocnemius & & \\
Lateral Gastrocnemius & & \\
\hline DAE = dynamic air exchange. &
\end{tabular}

when wearing the DAE prosthesis. Finally, subjects agreed $(6.4 \pm 3.1)$ that they had to stop and dry their residual limb when wearing the Suction prosthesis, but strongly disagreed $(0.8 \pm 0.7)$ with that statement when wearing the DAE prosthesis.

\section{DISCUSSION}

Accumulation of sweat inside the prosthetic socket of people with lower-limb amputation can lead to the prosthesis and liner sliding off or the users significantly reducing their activities so their prosthesis will stay on. The usual way to solve this problem is to simply doff the prosthesis, pour out the accumulated sweat, towel off the residual limb, and resume activity. The purpose of this study was to compare the performance of a novel prosthesis that can expel accumulating perspiration with a standard-of-care while participants performed a repeatable, laboratory-based protocol.

A limitation of this study was the small sample size $(n=5)$ of persons with transtibial amputation who were moderate community ambulators. Applying these results to the broader amputee population who have higher or lower mobility, different etiologies, or different amputation levels (e.g., transfemoral) should be done with caution.

The activity level of individuals while wearing the study prostheses was not statistically different, but this may be due to the large variation in activity levels. Importantly, the activity levels while wearing either prosthesis remained above the 1,100 to 1,450 prosthetic leg steps/d threshold for remaining a community ambulatory [20-21], suggesting that either study prosthesis would enable individuals to live independently. Unsolicited posttest comments by the subjects provide some insight regarding the large variability in the observed differences. One subject had difficulty aligning the locking pin while donning the DAE prosthesis and bruised the distal end of his residual limb. His frustration and discomfort may account for the observation that his daily step count while wearing the Suction prosthesis was more than three times the amount when wearing the DAE prosthesis. Another subject was moving to a new residence while wearing the Suction prosthesis and injured himself (not study related), which may explain why his activity level was more than four times the amount while wearing the DAE prosthesis as opposed to the Suction prosthesis. 
Another subject inquired about wearing the DAE prosthesis while running during the initial fitting. As we had not performed any standardized load tests (e.g., [22]), we asked the subject to only use the DAE prosthesis during low-impact athletic activities like walking and biking. Upon completion of the protocol, this subject admitted that he did not wear it to work because he worried it would not hold up to the rigors of his job, which may explain his very low activity level ( $<200$ prosthetic steps/ d) while wearing the DAE prosthesis in comparison to his activity level while wearing the Suction prosthesis ( $>4,000$ prosthetic steps/d).

The DAE prosthesis had negligible effects on residual-limb skin temperatures when compared with the Suction prosthesis. Despite the air flow produced by the small differential pressure between the proximal and distal ports of the DAE prosthesis, it was insufficient to reduce local skin temperatures. However, it is possible that operation of the DAE system produced some convective cooling that countered additional heat sourced by local vasodilation of the gastrocnemius muscle. Such a scenario might result in a lower core temperature while the local skin temperature remained constant. Heat sourced by friction from residual-limb pistoning may have also countered any convective cooling by the DAE, but this scenario would likely have no effect on core temperature. Skin temperatures could also have been influenced by the thicknesses and materials of socks worn between the liner and the socket on both study prostheses. Subjects were free to don socks during acclimation and prior to testing to achieve a satisfactory fit. The sockets for this study were fabricated following our clinical practice of between a 0 - and 2-ply fit. While in situ sock thickness is difficult to measure, we suggest the sock thickness, if present, was thin.

The DAE prosthesis was able to expel perspiration while maintaining secure adherence during the rest-walkrest protocol. Interestingly, the total amount of perspiration (accumulated plus expelled) while wearing the DAE was 80 percent more than the Suction prosthesis. If the approximately $0.66 \mathrm{~g}$ of perspiration expelled by the DAE had remained between the skin and liner, it is unknown whether that amount would have resulted in an insecure adherence. While a little moisture may increase the skin coefficient of friction [11-12], if sufficient perspiration accumulates to produce a thin film of moisture, the coefficient of friction can become greatly reduced. The time to loss of adherence under controlled conditions (e.g., activity intensity, environment temperature and humidity) would aid in defining perspiration thresholds for secure adherence.

The user experience was measured by the PEQ, modified such that subjects reported their experiences over a $1 \mathrm{wk}$ rather than a 4 wk period. The shorter duration may have weakened its psychometric properties by making it more difficult to distinguish effects between the subject's current prosthetic prescription and the study prostheses. The results suggest less frustration while wearing the Suction prosthesis but better residual-limb health and ambulation while wearing the DAE prosthesis. Subjects consistently reported less sweat inside their prosthesis, and over half reported less odor while wearing the DAE prosthesis. Two subjects were particularly displeased with the amount of sweat and odor inside their Suction prosthesis. In general, subjects reported maintaining good skin health with both systems. Unfortunately, one subject bruised the distal end of his residual limb while donning the DAE prosthesis and another developed a quarter-sized blister while wearing the Suction prosthesis during each subject's acclimation week. One subject experienced an abrasion from a thermistor while testing his first study prosthesis (DAE) and subsequently reported low scores regarding skin health while wearing the Suction prosthesis. It is unclear whether those scores reflect the prior existence of the abrasion or not. The results regarding frustration with the system were highly variable (large standard deviations), but in general the subjects thought the Suction prosthesis was less frustrating than the DAE prosthesis. The most frustrated user of the DAE prosthesis was the first subject, who found it difficult to align the pin with the locking mechanism. The pin and lock housing were modified, and we notified the subsequent subjects to be mindful of alignment and to be patient when donning the socket. Ambulation scores were similar for all questions except the ability to walk up stairs, on the street or sidewalk, and on surfaces, in which subjects generally had more confidence in the DAE prosthesis than the Suction prosthesis. One subject noted that the bulk of the suspension sleeve worn over the Suction prosthesis made ascending stairs difficult because of a limited range of knee motion.

The user experience was also measured by a custom questionnaire. The results suggest that the subjects generally perceived the DAE as more effective at providing thermal comfort and managing moisture accumulation than the Suction prosthesis. The subjects felt their residual 
limb was too hot and sweaty during activity while wearing the Suction compared with the DAE prosthesis, with over a six-point difference in scores. They also felt a greater need to stop and dry their residual limb while wearing the Suction than with the DAE, with a five-point difference in scores. This suggests the subjects perceived that the DAE prosthesis managed heat and moisture more effectively than the Suction prosthesis, a proposition supported by the subjects' opinion by a difference of over four points that the DAE kept the residual limb at a very comfortable temperature. The perception of adherence was highly variable (large standard deviations), but on average scored moderately low for both study prostheses. Interestingly, subjects opined they were more active than normal while wearing the DAE than the Suction prosthesis. This opinion is in contrast to the step activity results, but their positive perception suggests that they found the DAE prosthesis to be able to provide desirable benefits with an acceptable burden. A limitation of the custom questionnaire was that it only explored a few of the many domains related to satisfaction with a prosthesis.

\section{CONCLUSIONS}

This study found that a novel prosthesis was able to expel accumulating perspiration when people with lowerlimb amputation participated in a perspiration-inducing activity. In comparison with a standard-of-care prosthesis, the activity levels of the participants during a weeklong acclimation period were the same regardless of which prosthesis was worn, though the lack of statistical difference may have been due to circumstances and behavioral modifications that emerged from the sample population $(n=5)$. Residual-limb skin temperatures were also the same over the duration of a rest-walk-rest protocol that included a 30 min treadmill walk at self-selected speed while wearing thermally insulative garments. While no subject lost adherence during the laboratory test (both study prostheses retained $\sim 1 \mathrm{~g}$ of perspiration), the novel prosthesis expelled 38 percent of the total perspiration, suggesting it may improve adherence under more demanding conditions. Questionnaire results suggest the participants were receptive to both prostheses.

\section{ACKNOWLEDGMENTS}

\section{Author Contributions:}

Study concept and design: G. K. Klute, C. King.

Acquisition of data: K. J. Bates, J. S. Berge.

Analysis and interpretation of data: G. K. Klute, K. J. Bates,

J. S. Berge, W. Biggs, C. King.

Drafting of manuscript: G. K. Klute.

Critical revision of manuscript for important intellectual content:

G. K. Klute, K. J. Bates, J. S. Berge, W. Biggs, C. King.

Statistical analysis: K. J. Bates, J. S. Berge.

Obtained funding: G. K. Klute.

Study supervision: G. K. Klute.

Financial Disclosures: The authors have declared that no competing interests exist.

Funding/Support: This material was based on work supported by the Department of the Army, Advanced Prosthetics and Human Performance, U.S. Army Medical Research \& Materiel Command at the Telemedicine and Advanced Technology Research Center (grant W81XWH-11-2-0169) and the Department of Veterans Affairs (VA), Rehabilitation Research \& Development Service (awards A9186R, A9243C, and A4843C).

Additional Contributions: Since this work was completed, the first affiliation of authors G. K. Klute, K. J. Bates, J. S. Berge, and W. Biggs has changed to Center for Limb Loss Prevention and Prosthetic Engineering, VA Rehabilitation Research and Development, Puget Sound Health Care System, Seattle, Washington.

Institutional Review: This study was approved by the VA Puget Sound Health Care System Institutional Review Board and the U.S. Army Medical Research and Materiel Command, Office of Research Protections, Human Research Protection Office.

Participant Follow-Up: The authors have no plans to notify the study subjects of the publication of this article because of a lack of contact information.

\section{REFERENCES}

1. Legro MW, Reiber G, del Aguila M, Ajax MJ, Boone DA, Larsen JA, Smith DG, Sangeorzan B. Issues of importance reported by persons with lower limb amputations and prostheses. J Rehabil Res Dev. 1999;36(3):155-63.

\section{[PMID:10659798]}

2. Hagberg K, Brånemark R. Consequences of non-vascular trans-femoral amputation: A survey of quality of life, prosthetic use and problems. Prosthet Orthot Int. 2001;25(3): 186-94. [PMID:11860092] http://dx.doi.org/10.1080/03093640108726601

3. Meulenbelt HE, Geertzen JH, Jonkman MF, Dijkstra PU. Skin problems of the stump in lower-limb amputees: 2. Influence on functioning in daily life. Acta Derm Venereol. 2011;91(2):178-82. [PMID:21279299] http://dx.doi.org/10.2340/00015555-1023

4. Ghoseiri K, Safari MR. Prevalence of heat and perspiration discomfort inside prostheses: Literature review. J Rehabil 
JRRD, Volume 53, Number 6, 2016

Res Dev. 2014;51(6):855-68. [PMID:25356571]

http://dx.doi.org/10.1682/JRRD.2013.06.0133

5. Gholizadeh H, Abu Osman NA, Eshraghi A, Ali S, Razak NA. Transtibial prosthesis suspension systems: Systematic review of literature. Clin Biomech (Bristol, Avon). 2014; 29(1):87-97. [PMID:24315710]

http://dx.doi.org/10.1016/j.clinbiomech.2013.10.013

6. Hansen C, Godfrey B, Wixom J, McFadden M. Incidence, severity, and impact of hyperhidrosis in people with lowerlimb amputation. J Rehabil Res Dev. 2015;52(1):31-40.

[PMID:26230919] http://dx.doi.org/10.1682/JRRD.2014.04.0108

7. Peery JT, Ledoux WR, Klute GK. Residual-limb skin temperature in transtibial sockets. J Rehabil Res Dev. 2005;42(2): 147-54. [PMID:15944879] http://dx.doi.org/10.1682/JRRD.2004.01.0013

8. Klute GK, Berge JS, Huff E, Ledoux WR. The effect of rest and exercise on residual limb skin temperatures. Proceedings of the American Academy of Orthotists and Prosthetists Annual Meeting and Scientific Symposium; 2006; Chicago, IL.

9. Klute GK, Huff EA, Ledoux WR. In-socket skin temperatures and perception of comfort over a whole day. Proceedings of the Annual Meeting of the American Academy of Orthotists and Prosthetists; 2007; San Francisco, CA.

10. Shibasaki M, Wilson TE, Crandall CG. Neural control and mechanisms of eccrine sweating during heat stress and exercise. J Appl Physiol. 2006;100(5):1692-1701. [PMID:16614366] http://dx.doi.org/10.1152/japplphysiol.01124.2005

11. Gerhardt LC, Strässle V, Lenz A, Spencer ND, Derler S. Influence of epidermal hydration on the friction of human skin against textiles. J R Soc Interface. 2008;5(28):131728. [PMID:18331977] http://dx.doi.org/10.1098/rsif.2008.0034

12. Sivamani RK, Goodman J, Gitis NV, Maibach HI. Friction coefficient of skin in real-time. Skin Res Technol. 2003; 9(3):235-39. [PMID:12877684] http://dx.doi.org/10.1034/j.1600-0846.2003.20361.x

13. Adams M, Briscoe B, Johnson S. Friction and lubrication of human skin. Tribol Lett. 2007;26(3):239-53. http://dx.doi.org/10.1007/s11249-007-9206-0

14. Derler S, Gerhardt LC. Tribology of skin: Review and analysis of experimental results for the friction coefficient of human skin. Tribol Lett. 2012;45:1-27. http://dx.doi.org/10.1007/s11249-011-9854-y

15. Webber CM, Davis BL. Design of a novel prosthetic socket: Assessment of the thermal performance. J Bio- mech. 2015;48(7):1294-99. [PMID:25840507]

http://dx.doi.org/10.1016/j.jbiomech.2015.02.048

16. Ghoseiri K, Zheng YP, Hing LL, Safari MR, Leung AK. The prototype of a thermoregulatory system for measurement and control of temperature inside prosthetic socket. Prosthet Orthot Int. 2015. [PMID:26068464] http://dx.doi.org/10.1177/0309364615588343

17. Han Y, Liu F, Dowd G, Zhe J. A thermal management device for a lower-limb prosthesis. Appl Therm Eng. 2015;82:246-52.

18. Wernke M, Schroeder R, Haynes M, Kelly C, Colvin J. Management of skin temperature and perspiration using a modified liner. Proceedings of the American Academy of Orthotists and Prosthetists 41st Academy Annual Meeting and Scientific Symposium; 2015 Feb 18-21.

19. Legro MW, Reiber GD, Smith DG, del Aquila M, Larsen J, Boone D. Prosthesis evaluation questionnaire for persons with lower limb amputations: Assessing prosthesis-related quality of life. Arch Phys Med Rehabil. 1998;79(8):93138. [PMID9710165], http://dx.doi.org/10.1016/S0003-9993(98)90090-9

20. Holden JM, Fernie GR. Minimal walking levels for amputees living at home. Physiother Can. 1983;35(6):317-20.

21. Holden JM, Fernie GR. Extent of artificial limb use following rehabilitation. J Orthop Res. 1987;5(4):562-68.

[PMID:3681530]

http://dx.doi.org/10.1002/jor.1100050411

22. Prosthetics-Structural testing of lower-limb prosthesesRequirements and test methods. Geneva (Switzerland): International Organization for Standardization; 2016. ISO 10328:2006.

Submitted for publication June 29, 2015. Accepted in revised form December 17, 2015.

This article and any supplementary material should be cited as follows:

Klute GK, Bates KJ, Berge JS, Biggs W, King C. Prosthesis management of residual-limb perspiration with subatmospheric vacuum pressure. J Rehabil Res Dev. 2016;53(6):721-28.

http://dx.doi.org/10.1682/JRRD.2015.06.0121

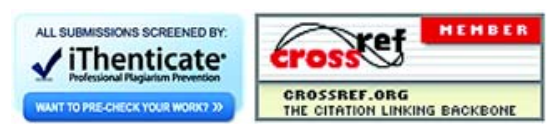

